\title{
Visualization and Segmentation of Cells in Unstained Paraffin-Embedded Cerebral Tissue.
}

Hans Deyhle $^{1,2, *}$, Christos Bikis ${ }^{1}$, Anna Khimchenko ${ }^{1}$, Gabriel Schweighauser ${ }^{3}$, Jürgen Hench ${ }^{3}$, Georg Schulz $^{1}$, Bert Müller ${ }^{1}$ and Simone E. Hieber ${ }^{1}$

${ }^{1 .}$ Biomaterials Science Center, Department of Biomedical Engineering, University of Basel, Allschwil, Switzerland.

2. X-ray Nanoimaging Group, School of Physics and Astronomy, University of Southampton, Southampton, United Kingdom.

3. Institute of Pathology, Department of Neuropathology, University Hospital of Basel, Basel, Switzerland.

**Corresponding author, hans.deyhle@unibas.ch

The three-dimensional visualization of cerebral cells is a challenging task. The human brain with a weight of about $1 \mathrm{~kg}$ contains around $10^{12}$ cells. Currently, brain cells are imaged by optical and electron microscopies. These imaging techniques yield only information on surface-near regions and volumes restricted to cubic micrometers. In the last decade, the tissue preparation and high-resolution hard X-ray tomography has been advanced to reveal individual unstained cells in cubic millimeter brain volumes [1,2] with phase contrast. More recently, sufficient contrast for cell imaging in single distance propagation mode [3] or even with lab-based conventional approaches [4] was obtained by embedding brain specimens in paraffin. This allows improving the spatial resolution from the regime of grating interferometry, limited by the grating period, usually in the range of a few micrometers, to subcellular length-scales. Such data lend themselves for (semi-)automatic cellular und subcellular structure segmentation. Several thousand Purkinje cells were visualized and segmented within a $\sim 40 \mathrm{~mm}^{3}$ cerebellum specimen. Individual dendritic trees of Purkinje cells were visualized from the same specimen with higher resolution scans.

The cerebellum was excised from a donated body of a 73-year old male in accordance with the Guidelines of the Ethical committee Northwestern Switzerland. It was embedded in paraffin following fixation in 4\% histological-grade buffered formalin and dehydration in ethanol. Two cylinders with diameters 2.6 and $6.0 \mathrm{~mm}$ were cut from the paraffin blocks by means of hollow punches. Singledistance propagation phase contrast micro computed tomography was performed at the beamlines ID19 (European Synchrotron Radiation Facility, Grenoble, France) and Diamond Manchester Imaging Branchline I13-2 (Diamond Light Source, Didcot, UK) in local mode. Measurements at ID19 were performed with pink beam with a mean photon energy of $19.45 \mathrm{keV}$ at $1.75 \mu \mathrm{m}$ pixel length. 2004 equiangular projections were acquired over $360^{\circ}$ with a FReLoN $2 \mathrm{~K}$ CCD camera with $1 \mathrm{~s}$ exposure per projection after $80 \mathrm{~cm}$ propagation distance. At I13-2, $19 \mathrm{keV}$ monochromatic beam energy and $0.45 \mu \mathrm{m}$ pixel length were used. Projections were acquired with a pco.4000 camera (PCO AG, Kelheim, Germany) with $8 \mathrm{~s}$ exposure time at 2400 equiangular steps over $180^{\circ}$ and $5 \mathrm{~cm}$ propagation distance. Phase retrieval was performed with the algorithm proposed [5] and implemented in the software package ANKAphase [6]. Reconstruction of the tomographic volumes was performed with the filtered backprojection routine implemented in MATLAB R2014b (Simulink, The MathWorks, Inc., USA). After tomographic measurements, the specimens were re-embedded into larger paraffin blocks for histological 
sectioning and subsequent staining with hematoxylin- and eosin-stain and digitized using a histological slide scanner (Olympus VS120 Virtual Slide Microscope, Japan), which served for data validation. The histological slices were then registered to the reconstructed volumes with a 2D-3D registration algorithm based on the work of Chicherova et al. [7].

The dataset recorded at ID19 allowed for the automatic cell counting of Purkinje. Here, a modification of Frangi-filtering was applied to segment the cells by identifying elliptically shaped objects in the specific size-range of interest. After manually setting the filter parameters, cell segmentation was performed in an automatic fashion. The error of cell localization was determined to be to $5 \%$ by visual inspection [3]. Purkinje cell density was found to be $116 \mathrm{~mm}^{-3}$, in agreement with values found in current literature [8]. The data also allows identifying subcellular structures in a three-dimensional fashion (cf. Figure 1). Here, three orthogonal slices through a Purkinje cells acquired at I13-2 are shown. This region with volume of $2.2 \times 10^{-4} \mathrm{~mm}^{3}$ was exemplarily extracted from the acquired $3.2 \mathrm{~mm}^{3}$ volume. The nucleolus, a compact structure composed mainly of RNA and proteins, is identified as dark circular feature in the center of the cell. The virtual cuts also represent parts of the dendritic tree.

The access to morphology with sub-cellular resolution through micro computed tomography has several advantages over established methods. Serial sectioning, besides being time consuming, is prone to artifacts like folding or tissue rupture or loss, and the specimen is unavailable for further processing. More sophisticated methods, like tissue clearing, are time consuming, technically demanding, and restricted to a limited amount of available stains. Contrary, $\mu \mathrm{CT}$ of paraffin-embedded specimens is fully compatible with the established histology and provides complementary information and helps to determine an optimized plane for sectioning. The segmentation pipeline presented allows automatically identifying Purkinje cells, and upscaling to larger volumes is straightforward. Due to these features, we expect $\mu \mathrm{CT}$ and dedicated data analysis to become a relevant method in the post-mortem examination of human tissues, especially for diagnostic purposes, nowadays termed 'digital histology'.

\section{References:}

[1] G Schulz et al, J. R. Soc. Interface 7 (2010), 1665

[2] M Töpperwien et al, Sci Rep 7 (2017) 42847

[3] SE Hieber et al, Sci. Rep. 6 (2016), 32156

[4] A Khimchenko et al, NeuroImage 139 (2016) 26

[5] D Paganin et al, J. Microsc. 206 (2002), 33

[6] T Weitkamp et al, J. Synchrotron Radiat. 18 (2011), 617

[7] N Chicherova et al in "Lecture Notes in Computer

Science, vol 8673", ed. P Golland et al, (Springer, Cham), 243

[8] J Skefos et al, PLoS ONE 9 (2014), e81255

[9] The project was supported by the ESRF proposal

MD-861) and supported by Swiss National Science

Foundation (SNSF) projects 147172 and 150164. The support by A Rack during measurements is gratefully acknowledged.

The support of staff at the Diamond Light Source, Didcot, UK, is highly appreciated.

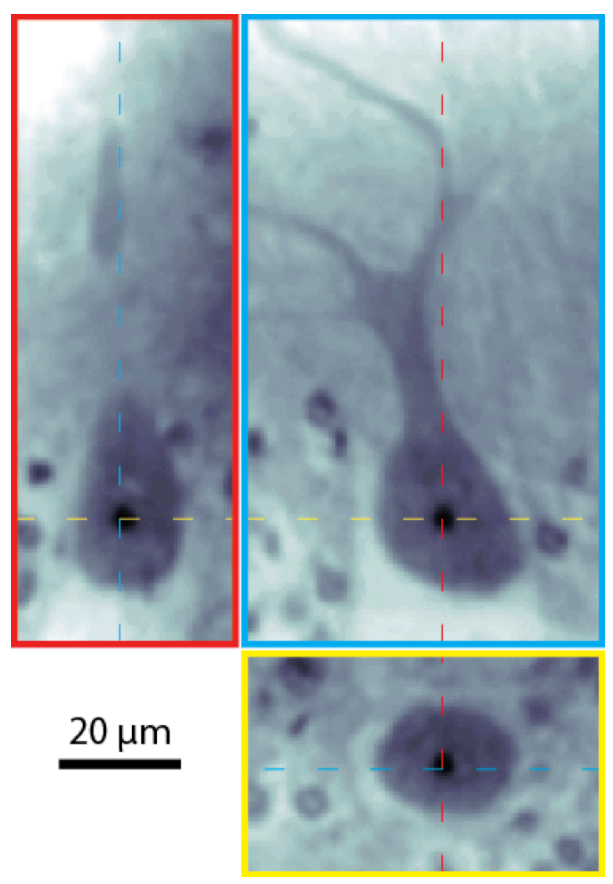

Figure. 1. Three orthogonal slices through a Purkinje cell, acquired at I13-2. The dark central spot corresponds to the nucleolus. 